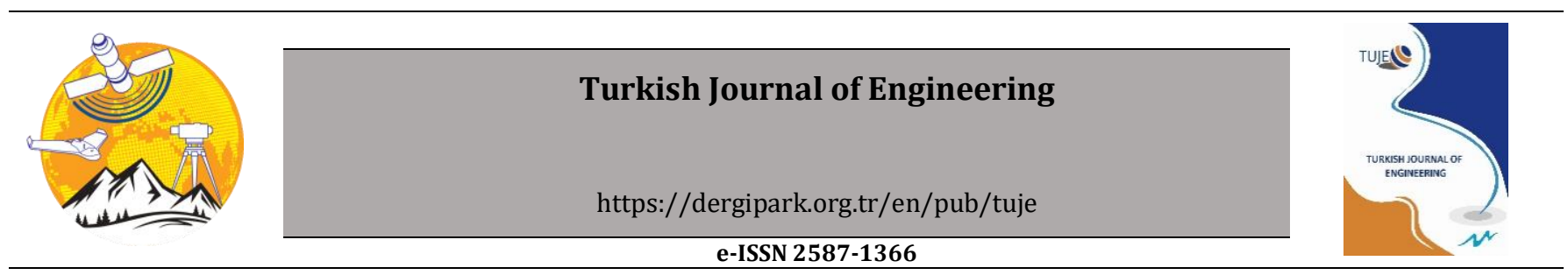

\title{
Graphene produced with using surfactant from expanded graphite
}

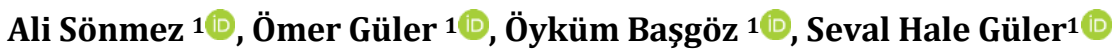 \\ ${ }_{1}^{1}$ Mersin University, Engineering Faculty, Department of Metallurgical and Materials Engineering, Mersin, Turkey
}

\author{
Keywords \\ Graphene \\ Ortho-dichlorobenzene \\ Expanded graphite \\ LPE
}

\begin{abstract}
Graphene is a single layer of graphite with hexagonal structure which have stacked layers. Graphene has recently been recognized by its researchers due to it have sp2 bonds in hexagonal lattice, the two-dimensional structure of a single atomic thickness, and its superior electrical, electrochemical, optical, thermal, mechanical properties and lightness. Liquid phase exfoliation (LPE) is the most promising method of graphene research to produce high quality graphene in the production of single or low layer graphene layers. In addition, LPE is an easy and inexpensive method as well as enables the production of higher capacity graphene. LPE method in the production of graphene is the use of surfactant mainly to make water suitable as a means of exfoliation. Furthermore, graphene adsorbable surfactants provide an effective charge by electrostatic repulsion to prevent the re-aggregation of graphene sheets, thus providing stabilization against the recombination of suspended graphene layers. Because 1,2Dichlorobenzene or ortho-dichlorobenzene (O-DCB) is both effective as solvent for graphene synthesis, it is one of the best production of graphene by the liquid phase exfoliation method. In this study, graphite powders were mixed in an acid solution of $\mathrm{H}_{2} \mathrm{SO}_{4}$ and $\mathrm{HNO}_{3}$ for $12 \mathrm{~h}$. The resulting powder was washed with distilled water until a neutral $\mathrm{pH}$ was obtained and then subjected to thermal treatment to obtain expanded graphite. The resulting powder was then mixed in an ultrasonic homogenizer in a mixture of O-DCB for $2 \mathrm{~h}$ using $50 \%$ strength and then it was examined by Transmission electron microscope (TEM) and X-ray photoelectron spectroscopy (XPS).
\end{abstract}

\section{INTRODUCTION}

Graphene is known as a surprising material in recent years due to its new features related to its twodimensional structure (Monajjemi 2017; Dhakate et al. 2011). Its intriguing properties such as high strength, an optical transmittance of $97,7 \%$, carriermobility as high as $200,000 \mathrm{~cm}^{2} \mathrm{~V}^{-1} \mathrm{~s}^{-1}$ at room temperature and a Young's modulus of $0.5-1 \mathrm{TPa}$, perfect electrical conductivity and high thermal conductivity make it promising for various applications (Arao et al. 2017). Due to these features of graphene, flexible devices, highfrequency transistors, energy storage and transducers, sensors, biomedical applications and the production of new generation composites have been demonstrated that graphene can be made easily (Wei and Sun 2015). Today, the most widely used methods in the production of graphene; mechanical exfoliation, chemical method (reduction of graphene oxide), chemical vapor deposition (CVD), epitaxial growth in silicon carbide, liquid phase exfoliation method (LPE), electrochemical exfoliation, the solvothermal method, arc discharge (Lee et al. 2019).

Commercialization of graphene-oriented applications is inevitable, but cost-effective and highquality serial production is needed (Xu et al. 2014). Since the first day it was found and applied, the liquid phase exfoliation method has been proposed as the most promising method of graphene research to produce high quality single or low layer graphene sheets in solvent dispersion forms. The main advantage of this process is a simple and sizable process in which pure graphite or expanded graphite is applied directly to a solvent process to weaken the Van Der Waals tensile forces between graphene interlayers (Durge et al. 2014). Many successes have been achieved in the production of graphene by liquid phase exfoliation method by using numerous solvent systems and suitable surfactants (Huo et al. 
2015). Although the Liquid Phase Exfoliation Method is a relatively easy and inexpensive method, the quality of the products produced is higher than the products produced by other methods (Zhu et al. 2013; Narayan and Kim 2015; Novoselov et al. 2004).

In this study, graphite powders were mixed in an acid solution of $\mathrm{H}_{2} \mathrm{SO}_{4}$ and $\mathrm{HNO}_{3}$ for $12 \mathrm{~h}$. The resulting powder was washed with distilled water until a neutral $\mathrm{pH}$ was obtained and then subjected to thermal treatment to obtain expanded graphite. The resulting powder was then mixed in an ultrasonic homogenizer in a mixture of 0 -DCB for $2 \mathrm{~h}$ using $50 \%$ strength. The main purpose of this study is to investigate the effects of O-DCB which is used as solvent in graphene synthesis by liquid phase exfoliation method in graphene production.

\section{EXPERIMENTAL PROCEDURE}

Hexagonal graphite powders were dried at $90{ }^{\circ} \mathrm{C}$ for 2 hours to remove moisture. The dried graphite powder was mixed for 12 hours in saturated acid containing $\mathrm{H}_{2} \mathrm{SO}_{4}$ and $\mathrm{HNO}_{3}$. After this process, this formed structure is called graphite intercalation compound (GIC). It was carefully washed with distilled water. Then, GIC was heated at $1000^{\circ} \mathrm{C}$ to form expanded graphite (EG).

The graphene layers are bound by weak van der Waals forces at some points of the EG and these bonds need to be broken. Therefore, EG was subjected to ultrasonication in O-DCB solvent. The suspension of 0.07 $\mathrm{mg} / \mathrm{ml}$ concentration of EG with $0-D C B$ was prepared. The solution of EG was kept at multi-frequency ultrasonication homogenizer for $1 \mathrm{~h}$. Then, the mixture was centrifuged at $5000 \mathrm{rpm}$ for 8 hours to remove 0DCB. The low density material suspended at the top layer of centrifuged solution was collected for further characterization. Transmission electron microscope (HRTEM) (JEOL Jem 1100) was used to investigate the microstructure of graphene samples. The samples were chractersized via X-ray photoelectron spectroscopy (XPS) (Specs-Flex) and Raman Spectroscopy (WITech alpha 300-R $512 \mathrm{~nm}$ wavelenght).

\section{RESULTS AND DISCUSSION}

The layers include a weak Van Der Waals bond. The aim of this study is to produce the graphene by breaking the Van Der Waals bonds between the graphite layers lined up. A solvent is required to separate the layers. Since the atomic diameters of the solvents that will allow the layers to separate are larger than the distance between the graphite layers, they cannot enter between the layers. For this reason, the distance between the layers of hexagonal graphite is first expanded by acid and thermal treatment. After these processes, the gap between the hexagonal layers widen and the weak bonds between the layers easily break through the incoming solvent atoms.

In previous our studies, different solvents (Such as DMF, NMP eg.) were used for graphene synthesis via Liquid Phase Exfoliation (LPE). It was shown in literature that O-DCB also is suitable a solvent because of its surface properties.

TEM images of samples were given after LPE process by using O-DCB as solvent. As can be seen in the figure, the exfoliation process was carried out successfully as planned and the layers were separated from each other. The width of the layers is over $200 \mathrm{~nm}$. In Figure 1a there is a dark colored fouling. Contamination is believed to be solvent residues during the production of amorphous carbon or graphene.
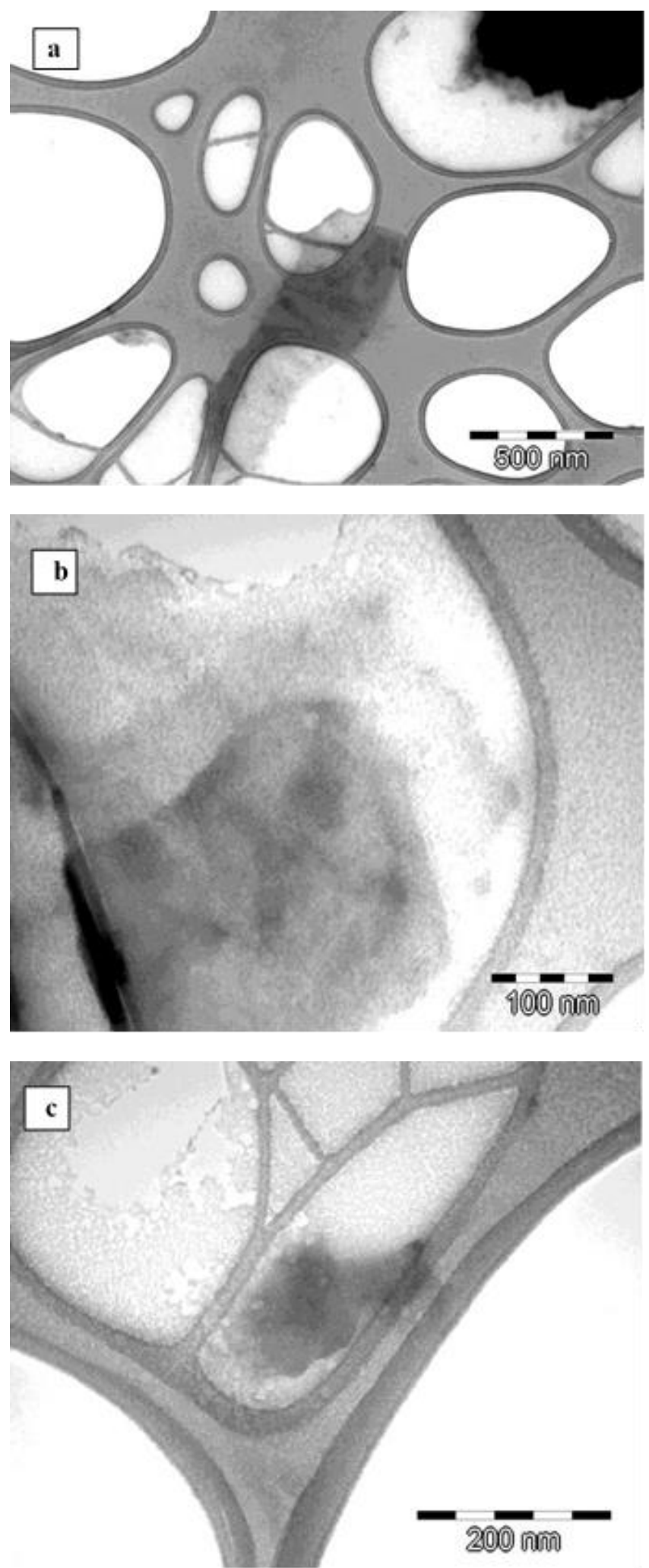

Figure 1. (a),(b),(c) TEM micrographs of high magnification of synthesized graphene layers

In Figure 2, XPS spectra analysis of samples were given. Figure 2a. shows general XPS spectra, Figure $2 \mathrm{~b}$. shows fitting of XPS peaks. As the figure shows that, 
synthesized graphenes contain C-C bonds over $80 \%$. In addition, $\mathrm{C}-\mathrm{O}$ bonds exist in samples. We think that $\mathrm{C}-\mathrm{O}$ bonds formed when EG was produced. Because, graphite powders were treated with acids and powders were heated to high temperature.
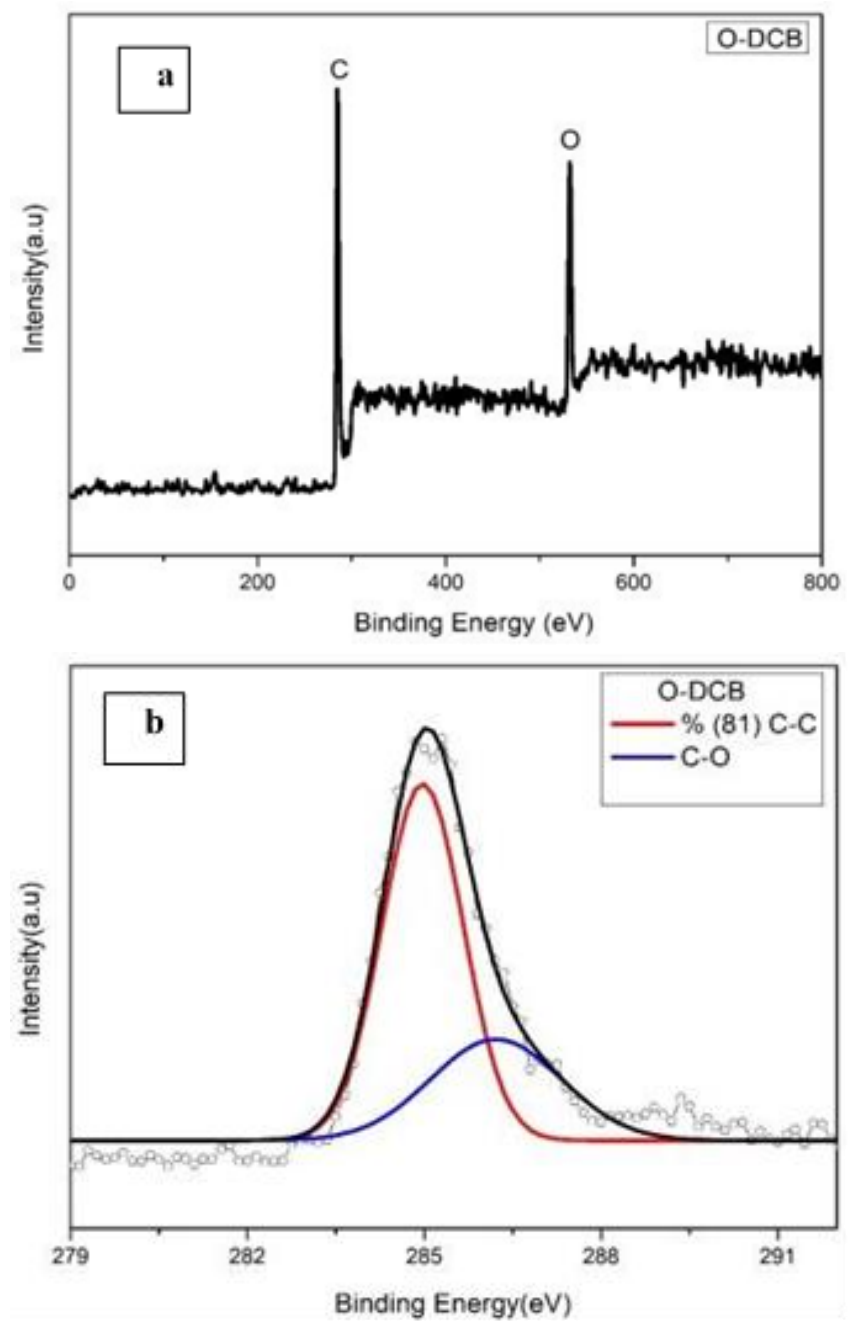

Figure 2. (a) General XPS spectra, (b) XPS spectra fitting of synthesized Graphene layers

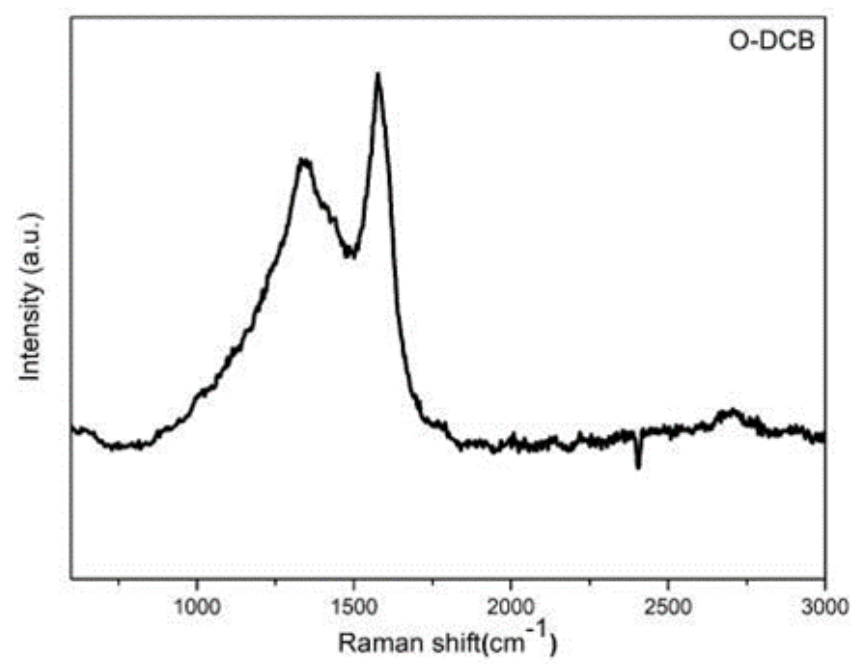

Figure 3. Raman Spectra of synthesized graphene layers

In this study, the analyzes show that production of graphene nanosheets were achieved with Liquid-Phase Exfoliation method by using O-DCB as solvent.
Figure 3 shows the Raman spectra of synthesized graphenes. In Raman spectra of graphenes, 3 characteristic peaks were shown. D peak (at $1350 \mathrm{~cm}^{-1}$ ) relate to defects in graphenes (in sheets basal plane or sheet edges). $\mathrm{G}$ peak (at $1582 \mathrm{~cm}^{-1}$ ) relates to $\mathrm{C}$ atoms vibrations having $\mathrm{sp}^{2}$ hibritazition in hexagonal lattice.

2D peak (at $2700 \mathrm{~cm}^{-1}$ ) indicates graphenes existence in powders. In Raman spectra of our samples, these peaks exist. The intensity of 2D peak is weak. This show that not only graphene exist in samples but also graphene nanosheets. The intensity of $\mathrm{D}$ peak is comparatively high. This show that defects exist in synthesized powders. We think that these defect formed during ultrasonic process.

\section{CONCLUSION}

In this study, 0-DCB was used as solvent in graphene synthesis via LPE method. In first step, EG was produced. Subsequently, EG was sonicated in O-DCB in order to synthesis graphene. It shown that graphene was synthesized by using O-DCB. But, graphene nanosheets also form together with graphene in samples.

\section{ACKNOWLEDMENT}

We would like to acknowledge the financial support from Mersin University Department of Scientific Research Projects (Project No: 2018-3-TP2-3085).

\section{REFERENCES}

Arao Y, Mori F \& Kubouchi M (2017). Efficient solvent systems for improving production of few-layer graphene in liquid phase exfoliation. Carbon, 118, 18-24. DOI: 10.1016/j.carbon.2017.03.002

Dhakate S R, Chauhan N, Sharma S, Tawale J, Singh S, Sahare P D \& Mathur R B (2011). An approach to produce single and double layer graphene from reexfoliation of expanded graphite. Carbon, 49(6), 1946-1954.DOI: 10.1016/j.carbon.2010.12.068

Durge R, Kshirsagar R V \& Tambe P (2014). Effect of sonication energy on the yield of graphene nanosheets by liquid-phase exfoliation of graphite. Procedia Engineering, 97, 1457-1465. DOI: 10.1016/j.proeng.2014.12.429

Huo C, Yan Z, Song X \& Zeng H (2015). 2D materials via liquid exfoliation: a review on fabrication and applications. Science Bulletin, 60(23), 1994- 2008. DOI:10.1007/s11434-015-0936-3

Lee X J, Hiew B Y Z, Lai K C, Lee L Y, Gan S, ThangalazhyGopakumar S \& Rigby S (2019). Review on graphene and its derivatives: Synthesis methods and potential industrial implementation. Journal of the Taiwan Institute of Chemical Engineers, 98, 163-180. DOI: 10.1016/j.jtice.2018.10.028

Monajjemi M (2017). Liquid-phase exfoliation (LPE) of graphite towards graphene: An ab initio study. Journal of Molecular Liquids, 230, 461- 472. DOI: 10.1016/j.molliq.2017.01.044

Narayan R \& Kim S O (2015). Surfactant mediated liquid phase exfoliation of graphene. Nano Convergence, 2(1), 20. DOI: 10.1186/s40580-0150050-x 
Novoselov K S, Geim A K, Morozov S V, Jiang D, Zhang Y, Dubonos S Vv et al. (2004). Electric field effect in atomically thin carbon films. Science, 306(5696), 666-669. DOI: 10.1126/science.1102896

Wei Y \& Sun Z (2015). Liquid-phase exfoliation of graphite for mass production of pristine few-layer graphene. Current Opinion in Colloid \& Interface Science, 20, 311-321. DOI: $10.1016 /$ j.cocis. 2015.10 .010
Xu J, Dang D K, Tran V T, Liu X, Chung J S, Hur S H, et al. (2014). Liquid-phase exfoliation of graphene in organic solvents with addition of naphthalene. Journal of Colloid and Interface Science, 418, 37-42. DOI: 10.1016/j.jcis.2013.12.009

Zhu L, Zhao X, Li Y, Yu X, Li C \& Zhang Q (2013). Highquality production of graphene by liquid phase exfoliation of expanded graphite. Materials Chemistry and Physics, 137(3), 984-990. DOI: 10.1016/j.matchemphys.2012.11.012

(C) Author(s) 2021.

This work is distributed under https://creativecommons.org/licenses/by-sa/4.0/ 